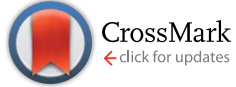

Cite this: J. Anal. At. Spectrom., 2014, 29,1714

Received 17th April 2014

Accepted 17th June 2014

DOI: 10.1039/c4ja00126e

www.rsc.org/jaas

\title{
Comprehensive analysis of metalloporphyrins via high irradiance laser ionization time-of-flight mass spectrometry
}

\author{
Zhibin Yin, ${ }^{a}$ Binwen Sun, ${ }^{a}$ Xiaohua Wang, ${ }^{a}$ Xiaoling Cheng, ${ }^{a}$ Wei Hang ${ }^{\star a b}$ \\ and Benli Huang ${ }^{a}$
}

\begin{abstract}
High-irradiance laser ionization time-of-flight mass spectrometry (LI-TOFMS), an established technique for elemental determination, has been applied for the analysis of metalloporphyrins. Many porphyrins and their metal complexes, being organometallic compounds, are hard to dissolve in general organic solvents, hampering the wider application of traditional mass spectrometric techniques. With LI-TOFMS, an environmentally friendly analytical strategy has been demonstrated, which is capable of matrix- and solvent-free analysis of metalloporphyrins, with advantages including direct solid sampling, ease of implementation, and avoidance of sample pre-treatment. Moreover, information about elemental composition, fragments, and intact molecules can be obtained simultaneously using LI-TOFMS, hence expediting the identification of metalloporphyrins. A comparative study of $\mathrm{LI}$-, laser desorption ionization (LDI-), matrix-assisted laser desorption ionization (MALDI-) and electrospray ionization (ESI-) mass spectrometry (MS) has also been conducted.
\end{abstract}

\section{Introduction}

Considerable effort has been devoted to the investigation of metalloporphyrins. These molecules consist of an electrondelocalized tetrapyrrolic unit with one incorporated metal ion. Insight into their unique chemical and physical properties accompanies the great interest being shown in a wide variety of applications, such as in catalysis, ${ }^{\mathbf{1 , 2}}$ solar cells, ${ }^{\mathbf{3}, \mathbf{4}}$ and petrochemistry. ${ }^{5}$ Aside from these artificially synthesized metalloporphyrins, many naturally occurring metalloporphyrins, especially magnesium and iron derivatives of porphyrins, are also indispensable for living organisms. Magnesium porphyrins, e.g. chlorophyll, play a critical role in light-harvesting and energy-transfer processes. Iron porphyrins, constituting the prosthetic groups in haemoglobin, myoglobin, and cytochromes, are involved in oxygen transport and storage as well as electron transfer. Compared to synthetic work, more attention needs to be given to the comprehensive analysis of these species.

Several mass spectrometric techniques, such as chemical ionization, ${ }^{5}$ electron ionization, ${ }^{6}$ MALDI- ${ }^{7}$ ESI- ${ }^{8-10}$ and secondary ion (SI-) MS, ${ }^{\mathbf{1 1}, \mathbf{1 2}}$ have been used to analyze porphyrins and their metal complexes. Many of these compounds, however,

${ }^{a}$ Department of Chemistry, MOE Key Lab of Spectrochemical Analysis \& Instrumentation, College of Chemistry and Chemical Engineering, Xiamen University, Xiamen, China

${ }^{b}$ State Key Laboratory of Marine Environmental Science, Xiamen University, China. E-mail:weihang@xmu.edu.cn are practically insoluble in water or general organic solvents other than toxic solvents. ${ }^{\mathbf{1 3}}$ Solvent- and matrix-based sample preparation generates contaminants and interference, which have hampered the wider application of MS for the analysis of these species. Furthermore, with conventional soft ionization sources, the aforementioned methods can scarcely acquire the information about metal elements of interest. Therefore, an environmentally friendly and rapid analytical strategy, in which the molecular and elemental data are acquired simultaneously, should be put forward for the unambiguous identification of metalloporphyrins.

LI-TOFMS has received a significant amount of attention in the direct elemental analysis of solids. ${ }^{\mathbf{1 4 1 5}}$ Of particular interest is that LI-TOFMS has been applied to the direct multi-elemental analysis of biological analytes, e.g., tea leaf, pig skin, and single cells. ${ }^{16,17}$ LI-TOFMS may also be considered for analysis of organic compounds, as this technique was used to successfully characterize benzoylferrocene. ${ }^{18}$ In this report, we focus on an LI-TOFMS analytical strategy, different from those methods mentioned, ${ }^{5-10}$ for the analysis of metalloporphyrins. Comprehensive information on metallic/nonmetallic elements, characteristic fragments, and intact molecules of analytes can be selectively acquired through the variation of helium buffer gas pressure in the ion source. Remarkably, the analyte can be analyzed straightforwardly by LI-TOFMS in its primary solid form, using a matrix-free and solvent-free analytical strategy, without tedious sample preparation. In addition to LI-MS, additional MS techniques, such as ESI-, MALDI- and LDI-MS, are compared with regards to the analysis of metalloporphyrins. 


\section{Experimental}

\section{Chemicals}

Cobalt tetramethoxyphenylporphyrin (CoTMPP, >96\% purity, ave. $M_{\mathrm{W}}=791.76 \mathrm{u}$ ) was purchased from J\&K Scientific Ltd (Beijing, China). Zinc meso-tetraphenylporphyrin (ZnTPP, $M_{\mathrm{W}}=678.11 \mathrm{u}$ ) was purchased from Alfa Aesar Co., Inc. and hemin $\left(M_{\mathrm{W}}=651.94 \mathrm{u}\right)$ was purchased from Solarbio Bioscience \& Technology Co., Ltd (Beijing, China). The high purity helium gas (99.999\%) was purchased from Linda Gas Co. Ltd (Xiamen, China).

\section{Instruments}

The LI experiments were carried out with an in-house-built LI-TOFMS, which was described in our previous articles. ${ }^{19}$ Briefly, a $532 \mathrm{~nm} \mathrm{Nd:YAG} \mathrm{laser} \mathrm{(NL303G,} \mathrm{EKSPLA)} \mathrm{with} 4.4 \mathrm{~ns}$ pulse duration and $5 \mathrm{~Hz}$ repetition rate was applied in the experiments; and the focal spot on the analyte surface was about $45 \mu \mathrm{m}$ in diameter. High-purity helium (99.999\%) was used as the buffer gas in the ionization chamber in order to cool the energetic ions and reduce the multiply charged ions. ${ }^{20}$ The gas pressure in the ionization chamber was controlled by a needle valve. The output signal from the microchannel plate was collected using a digital storage oscilloscope (42Xs, Lecroy, USA) with a $2 \mathrm{Gs} \mathrm{s}^{-1}$ sampling rate. Subsequently, an in-housecompiled program written in LabVIEW 8.0 was used for data processing. The mass accuracy of the instrument is about 200 ppm.

A high-resolution ESI-MS (micrOTOF-QII, Bruker Daltonics, USA) was operated in the positive-ion mode. In the source, the capillary voltage and the end plate offset potential were set at $-4500 \mathrm{~V}$ and $-500 \mathrm{~V}$, respectively. The nebulizer gas pressure was set at 0.8 bar and the dry gas flow rate was set at $2.0 \mathrm{~L} \mathrm{~min}^{-1}$ with a temperature of $180{ }^{\circ} \mathrm{C}$. Subsequently, CID MS/MS experiments were used for obtaining the dissociation products of the analytes. Argon was chosen as the collision gas with the collision energy ranging from 0 to $80 \mathrm{eV}$.

A commercial MALDI-TOFMS (microFlex, Bruker Daltonics) was used in the experiments. A $337 \mathrm{~nm}$ nitrogen laser with a pulse width of $5 \mathrm{~ns}$ was used; and laser irradiance was $7.0 \times 10^{7}$ $\mathrm{W} \mathrm{cm}^{-2}$. Reflection and positive-ion mode with an accelerating potential of $20 \mathrm{kV}$ was employed in the experiments. All the MALDI spectra were acquired from the accumulation of 100 laser shots.

As for LDI experiments, the same MALDI instrument with 2.0 $\times 10^{8} \mathrm{~W} \mathrm{~cm}^{-2}$ laser irradiance was used for the analysis of the analytes without any matrix assistance.

\section{Sample preparation}

For LI-MS experiments, all the above samples were in the form of powder and were dried in a vacuum drying oven for one hour before analysis. Subsequently, the powder samples were pressed into a disc by a hydraulic press machine under a pressure of $1 \times 10^{7} \mathrm{~Pa}$ for 5 minutes. The powder-pressed samples with a thickness of $0.5 \mathrm{~mm}$ and diameter of $6 \mathrm{~mm}$ were fixed on a homemade direct insertion probe with Kapton tape.
The stock solutions of CoTMPP and ZnTPP were prepared in chloroform, whereas hemin was dissolved in a mixture of dimethyl sulfoxide and $0.5 \%$ formic acid. The sample solutions were diluted stepwise 10 - to 1000 -fold to a concentration of $1 \times 10^{-5} \mathrm{~mol} \mathrm{~L}^{-1}$ for the ESI experiments.

For the MALDI experiments, the saturated solution of alphacyano-4-hydroxycinnamic acid (CHCA) was prepared using a mixture of solvents, comprising water-acetonitrile in a 30/70 (v/ v) ratio and containing $0.5 \%$ formic acid. The sample solution was mixed with the matrix solution, and a final concentration of $1 \times 10^{-5} \mathrm{~mol} \mathrm{~L}^{-1}$ was used for the MALDI experiments. The standard "dried droplet" method, in which $1 \mu \mathrm{L}$ of sample solution and $1 \mu \mathrm{L}$ of saturated matrix solution are mixed, was employed to prepare MALDI targets. ${ }^{21}$

Two $\mu \mathrm{L}$ of the sample solution with a concentration of $1 \times 10^{-2} \mathrm{~mol} \mathrm{~L}^{-1}$ was directly dropped on the MALDI plate for LDI experiments.

\section{Results and discussion}

A typical and fragment-rich mass spectrum of ZnTPP with very little interference is shown in Fig. 1a. In addition to information about the analyte's molecular weight, the metal element of interest as well as nonmetallic elements, e.g. C, H, and N, which are hard to detect by MALDI-MS, LDI-MS and ESI-MS, can be easily detected by LI-MS. A combination of molecular and elemental information can be of benefit for structural identification of metalloporphyrins. For comparison, spectra of ZnTPP using ESI-, MALDI-, and LDI-MS are depicted in Fig. 1b-d. Interference induced by the presence of solvents and matrices within the low-mass range for the ESI and MALDI (Fig. 1b and c) complicates the interpretation of the spectra. For LDI, high laser irradiance $\left(2.0 \times 10^{8} \mathrm{~W} \mathrm{~cm}^{-2}\right)$ is needed to acquire the molecular signal. It generates ions of high kinetic energy, which are unbearable for the on-axis TOF mass analyzer. The parent

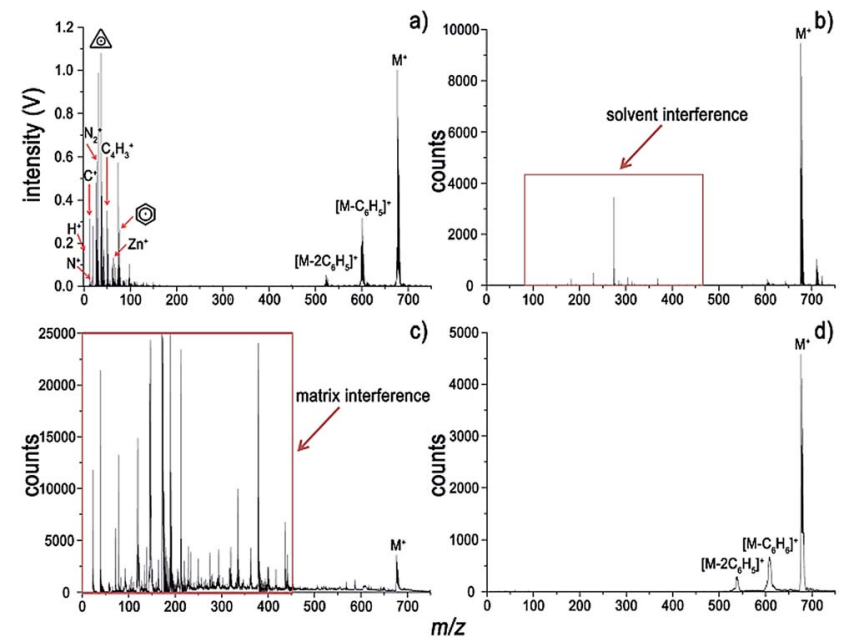

Fig. 1 Mass spectra of ZnTPP acquired using (a) LI-MS with $7.2 \times 10^{9}$ $\mathrm{W} \mathrm{cm}{ }^{-2}$ of laser irradiance at a helium pressure of $10 \mathrm{~Pa}$, (b) ESI-MS using chloroform as the solvent, (c) MALDI-MS with CHCA as the matrix, and (d) LDI-MS 
ion and some fragment peaks are observed at the expense of resolution. In contrast, the matrix- and solvent-free LI-MS strategy has the advantages of generating a clean background and fragment-rich information due to its direct sampling and ionization feature. In other words, complete information about the sample is contained in the LI spectrum without interference.

Very intense molecular ion and protonated parent ion peaks are observed for ZnTPP in Fig. 1a. Prominent molecular fragments arose from loss of $\mathrm{C}_{6} \mathrm{H}_{4}$ (that is $\left[\mathrm{M}+\mathrm{H}-\mathrm{C}_{6} \mathrm{H}_{5}\right]^{+}$), $\mathrm{C}_{6} \mathrm{H}_{5}$, $\mathrm{C}_{6} \mathrm{H}_{6}$ and $\mathrm{C}_{12} \mathrm{H}_{10}$. Moreover, loss of $\mathrm{H}$ and loss of $2 \mathrm{H}$ from the parent ion are observed in the LI spectrum. The loss of benzene can be ascribed to the high stability of the aromatic cyclic system. Given that many $\mathrm{H}$ atoms on the pyrrole rings adjacent to phenyl substituents, it can be postulated that the loss of benzene arose from the recombination between phenyl substituents and adjacent $\mathrm{H}$ atoms. The dominant molecular fragment at an $\mathrm{m} / \mathrm{z}$ of 522 is the result of the loss of $\mathrm{C}_{12} \mathrm{H}_{10}$. The strong preference for the loss of $\mathrm{C}_{12} \mathrm{H}_{10}$ might be attributed to the recombination of two $\mathrm{C}_{6} \mathrm{H}_{5}$ radicals, which is favored thermodynamically. ${ }^{8}$ Nevertheless, all the spectra in Fig. 1 reveal a loss of up to two phenyl substituents. Considering that ZnTPP is a large conjugated system with electronic delocalization, the loss of too many phenyl groups might be destabilizing. Additionally, much information on elements and fragments of ZnTPP can be acquired from the low-mass range of LI-MS (Fig. 1a) in contrast to ESI, MALDI and LDI as seen in Fig. 1b-d.

Traditional ESI tandem mass spectrometry (ESI/MS/MS), with electron-induced dissociation ${ }^{9,22}$ or collision-induced dissociation (CID), ${ }^{\mathbf{8} 23}$ is a powerful analytical tool for the elucidation of molecular structures. For comparison, the LI pressure-gradient spectra and the CID product ion spectra of ZnTPP are shown in Fig. 2. At first glance, the dominant fragment peaks in the LI spectra (Fig. 2a) and CID product ion spectra (Fig. 2b) appear to be similar in the high-mass range. Nevertheless, dramatic differences are evident between the two methods. Unlike the CID MS/MS technique, LI corresponds to the single-stage MS, but is capable of acquiring information about elemental composition, fragments, and intact molecules simultaneously. At low helium pressure (Fig. 2a), the spectra are dominated by intact molecular ions, characteristic fragments, and nonmetallic elemental ions. As pressure increases, the intensities of these ions become weaker, and the metal ions become the dominant species in the spectra. Comparing the spectra in Fig. 2a and b, the information about molecules and fragments in the CID MS/MS spectra can also be obtained in the LI single-stage spectra. Nevertheless, the metal ions sequestered in metalloporphyrins, nonmetallic elements, and the characteristic fragments within the low-mass range $(m / z<100)$ are unavailable from the CID MS/MS spectrum but acquired readily using LI-MS.

The surface of the solid analyte sample that is irradiated with a high-intensity laser undergoes rapid vaporization. After the phase transition, an opaque plasma is formed within several picoseconds. ${ }^{24}$ Intense atomization and ionization processes occur in the plasma whose temperature is over $10000 \mathrm{~K}$, yielding an abundant supply of energetic free electrons and

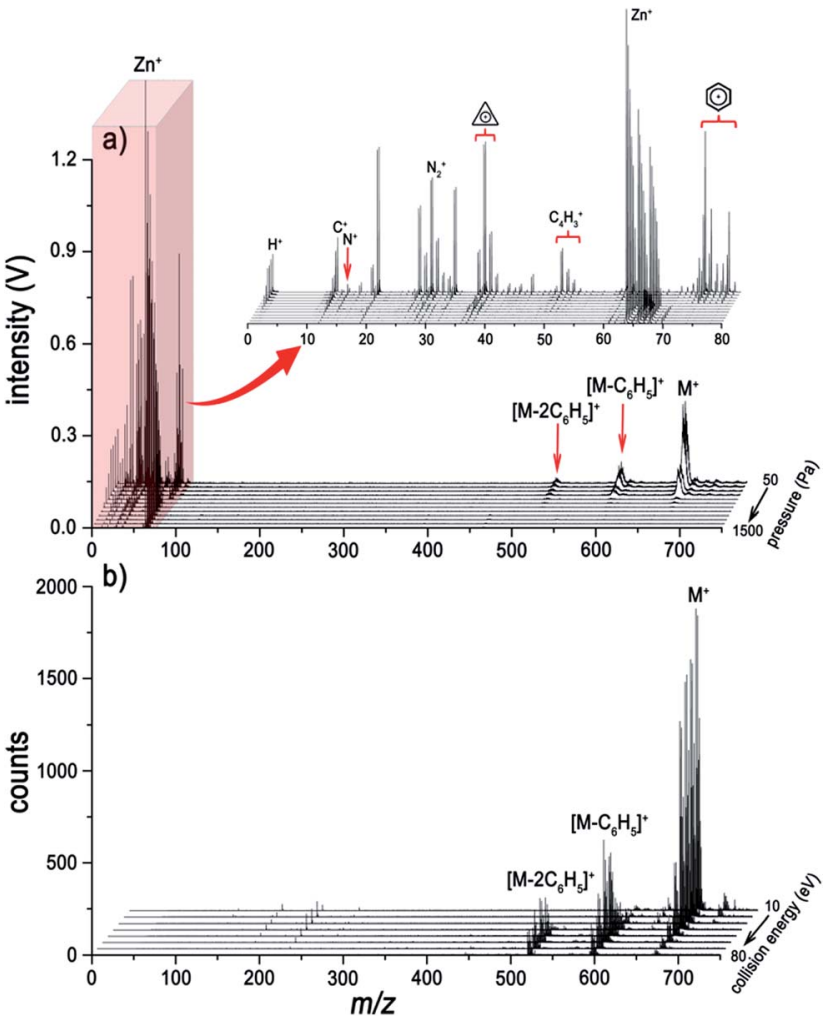

Fig. 2 (a) Typical pressure-gradient LI-mass spectra of ZnTPP with an applied laser irradiance of $7.2 \times 10^{9} \mathrm{~W} \mathrm{~cm}^{-2}$. (b) The dissociation gradient spectra of ZnTPP using ESI/MS/MS, in the form of CID, over the energy range from $10 \mathrm{eV}$ to $80 \mathrm{eV}$.

protons. ${ }^{25}$ In such a harsh plasma environment, no molecules and fragments can survive. However, it would appear that laser energy absorbed by the solid generates heat that dissipates into the surrounding area of the crater, where thermal diffusion desorption of the molecules will occur immediately. At low helium pressure, the dominant species in the spectra are molecular ions due to the long mean free paths (MFPs) of electrons and protons. We think that the molecular ions are produced through abundant collisions between the thermally desorbed molecules and high-energy electrons; and protonated molecular ions can also be produced by protonation as shown in Fig. 3. Alternatively, at high pressure, metal ions are generated in the hot and dense plasma, while no molecular information can be acquired because the electron impact and protonation processes rarely occur due to the short MFPs of electrons and protons.

To make our discussion more concrete, CoTMPP and hemin were chosen for analysis. The comparisons of the analysis of CoTMPP using LI-, ESI-, MALDI-, and LDI-MS are shown in Fig. 4 and the results for hemin are shown in Fig. 5. They exhibit similar trends as does ZnTPP in Fig. 1a, but the characteristic fragments are slightly different. As shown in Fig. 4a, the characteristic fragments arose from losses of $n \mathrm{CH}_{3}(n=1$ to 4$)$ and $\mathrm{C}_{6} \mathrm{H}_{4}\left(\mathrm{OCH}_{3}\right)_{n}(n=1$ to 4$)$ and can be observed clearly. And dramatic differences can be observed within the low-mass range $(m / z<100)$ in Fig. $4 a-d$. In contrast to the interference induced 


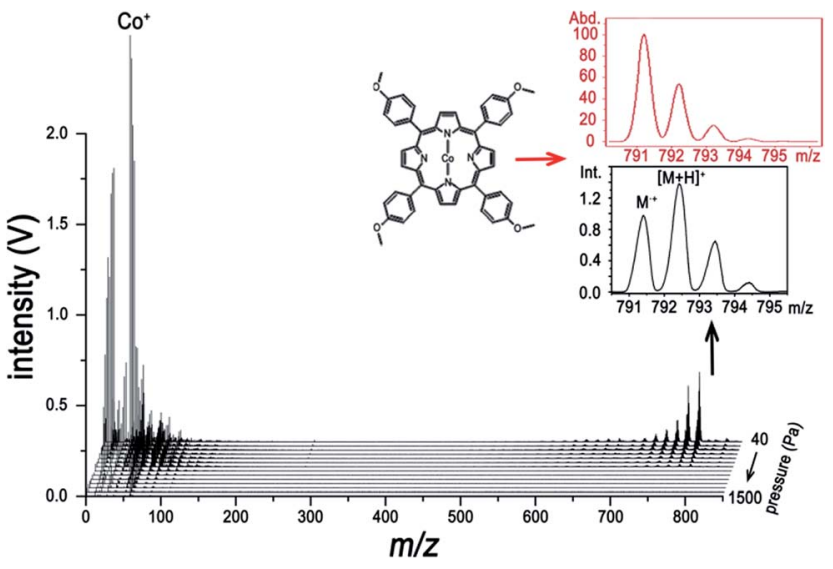

Fig. 3 Evidence for the protonated molecular ion $[\mathrm{M}+\mathrm{H}]^{+}$in the LI-TOFMS analysis of CoTMPP with laser irradiance at $9.3 \times 10^{9} \mathrm{~W} \mathrm{~cm}^{-2}$. The insets show the contrast between the theoretical isotope distribution (in red) and the acquired isotope distribution (in black).

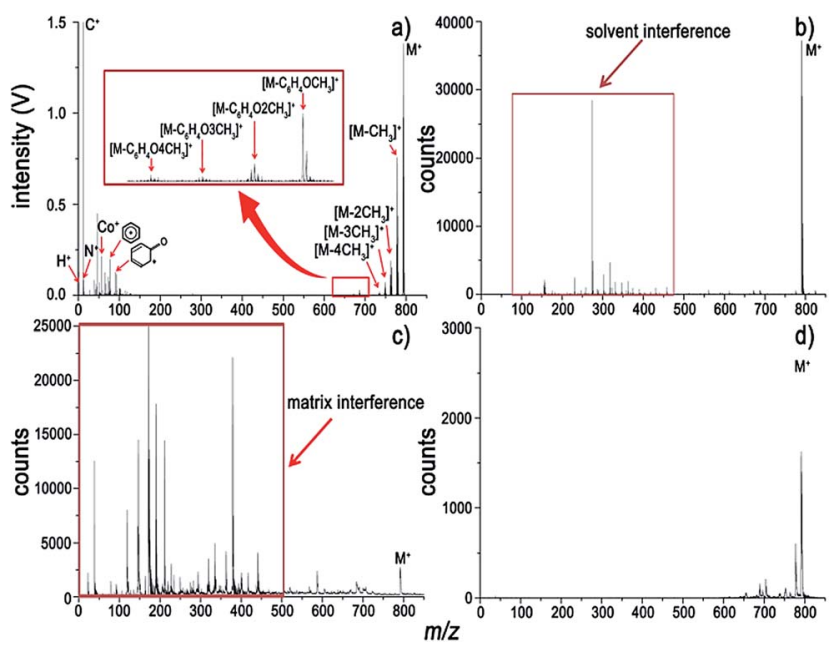

Fig. 4 Mass spectra of CoTMPP acquired using (a) LI-MS with $9.3 \times 10^{9} \mathrm{~W} \mathrm{~cm}^{-2}$ of laser irradiance at a helium pressure of $10 \mathrm{~Pa}$, (b) ESI-MS with chloroform as the solvent, (c) MALDI-MS with CHCA as the matrix, and (d) LDI-MS.

by solvent and matrix in Fig. $4 \mathrm{~b}$ and c, all the elements involved in the analyte, such as $\mathrm{H}, \mathrm{C}, \mathrm{N}$ and $\mathrm{Co}$, can be detected without interference by LI-MS (Fig. 4a). Similarly, simultaneous acquisition of elemental, fragmental and molecular information of hemin is also shown in Fig. 5a. Comparison with LI pressuregradient spectra and CID product ion spectra of hemin in Fig. 6 show easily observed differences within the low-mass range $(\mathrm{m} / \mathrm{z}<100)$ (Fig. 6a). All the elements involved in the analyte, such as $\mathrm{H}, \mathrm{C}, \mathrm{N}, \mathrm{O}$, and Fe, can be detected by LI-MS. The characteristic fragments can be observed clearly, which is of paramount importance for structure elucidation.

The fragment-rich spectra of hemin acquired using LI-MS are shown in Fig. 7. It is worth noting that all substituent groups can be detected in the LI spectra, which is rarely achieved using other MS methods. The spectrum yields information on all the

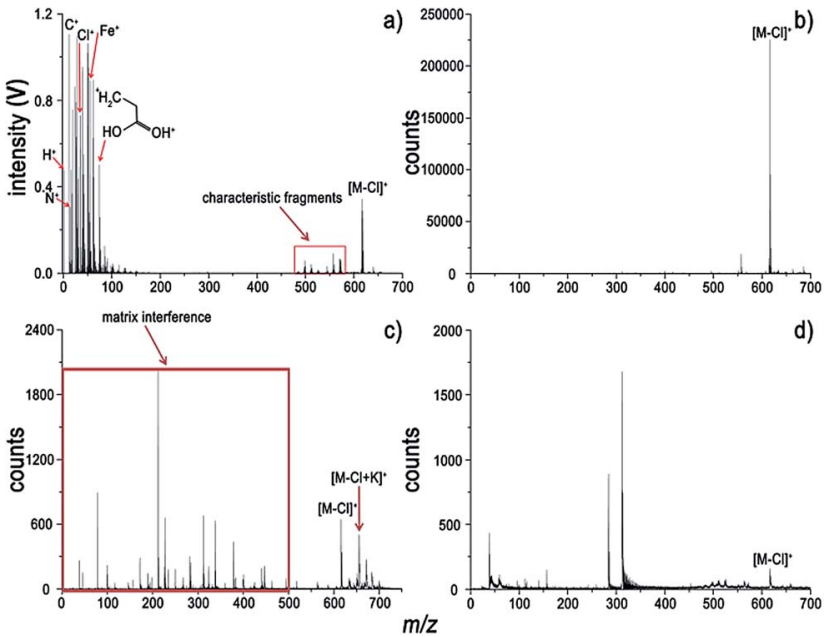

Fig. 5 Mass spectra of hemin from (a) LI-MS with $6.1 \times 10^{9} \mathrm{~W} \mathrm{~cm}^{-2}$ of laser irradiance at a helium pressure of $20 \mathrm{~Pa}$, (b) ESI/MS using dimethyl sulfoxide as the solvent (c) MALDI-MS with CHCA as the matrix, and (d) LDI-MS.

fragments, which is an essential prerequisite for the comprehensive analysis of intricate metalloporphyrins. The dominant molecular peak of the spectrum, seen in Fig. 7a, corresponds to the singly charged $[\mathrm{M}-\mathrm{Cl}]^{+}$at $m / z$ 616. In comparison with

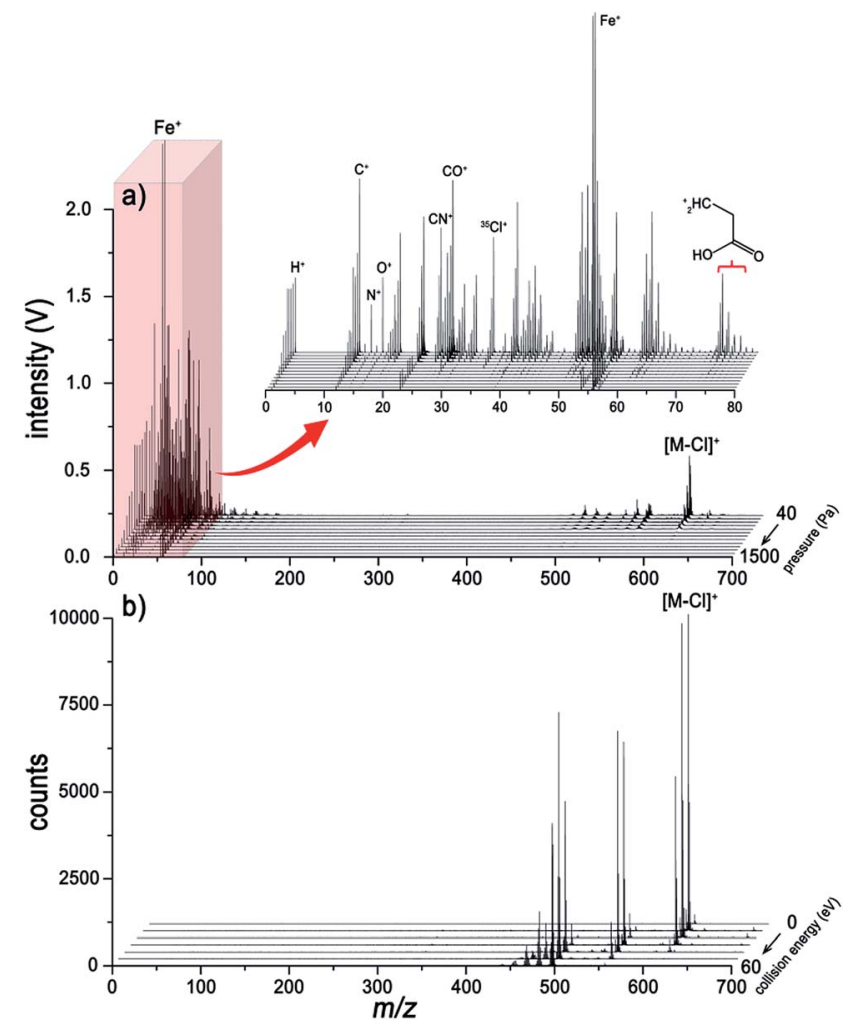

Fig. 6 (a) Typical pressure-gradient mass spectra of hemin acquired from LI-MS with laser irradiance of $6.1 \times 10^{9} \mathrm{~W} \mathrm{~cm}^{-2}$. The inset depicts an expanded region within an $\mathrm{m} / \mathrm{z}$ range of $0-80 \mathrm{u}$. (b) The dissociation gradient spectra of ZnTPP using ESI/MS/MS, in the form of CID with the energy range of $0-60 \mathrm{eV}$. 


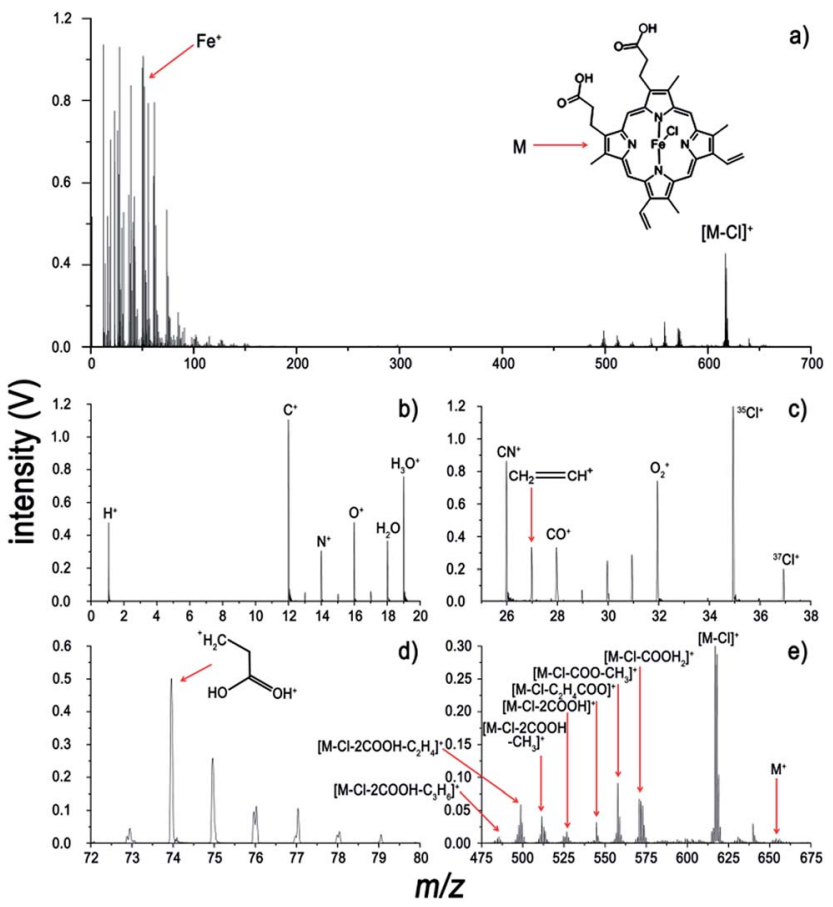

Fig. 7 Fragment-rich spectra of hemin from LI-TOFMS with laser irradiance of $6.1 \times 10^{9} \mathrm{~W} \mathrm{~cm}^{-2}$ at a helium pressure of $40 \mathrm{~Pa}$. (a) The spectrum in the $\mathrm{m} / \mathrm{z}$ range $0-700 \mathrm{u}$ and expanded views at (b) $0-20$, (c) 25-38, (d) 72-80, and (e) 475-675 u.

molecular fragments produced by ESI-MS/MS (see Fig. 6b) and LDI (see Fig. 5d), the elemental composition and all the substituents in the low-mass range can be acquired completely using LI-MS. This technique will contribute to further characterization of metalloporphyrins.

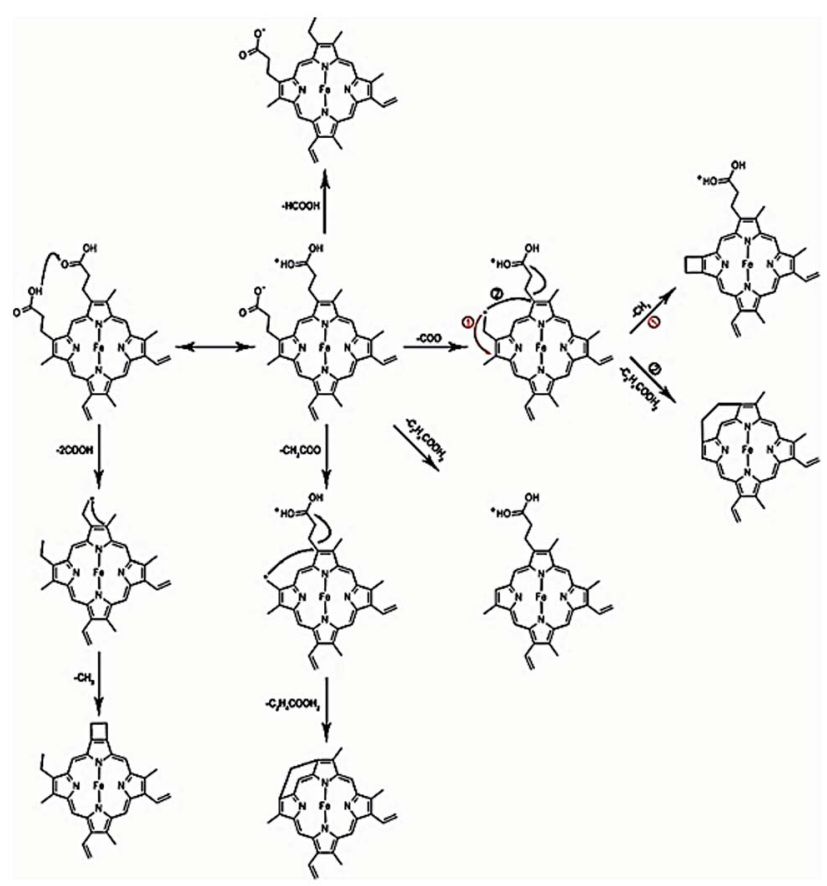

Scheme 1
The fragmentation pathways of hemin and mechanisms accounting for the production of the characteristic fragments are shown in Scheme 1. The primary fragment peak at $\mathrm{m} / \mathrm{z} 570$ corresponds to the loss of formic acid $(\mathrm{HCOOH})$ from hemin, and would appear to require a proton transfer between the two adjacent carboxyl groups of hemin prior to the fragmentation. Subsequently, the fragment peaks corresponding to an $\mathrm{m} / \mathrm{z}$ of 557 and $\mathrm{m} / \mathrm{z}$ of 498 can be attributed to fragments resulting from the loss of $\left(\mathrm{COO}^{-}+\mathrm{CH}_{3}\right)$ and of $\left(\mathrm{COO}^{-}+\mathrm{C}_{2} \mathrm{H}_{4} \mathrm{COOH}_{2}\right)$ respectively, which are abundant enough to be part of significant fragmentation pathways. It is anticipated that the particular stability of these two peaks is due to the intramolecular rearrangement pathways, along with the formation of four- and seven-membered rings. The stabilities of fragments at $\mathrm{m} / z 511$ and $m / z 485$, which involve formation of four-membered and six-membered rings, can also be explained by considering that cyclization is favoured thermodynamically. In addition, there is a distinct peak at $m / z 526$, which arises from loss of two carboxyl groups.

\section{Conclusions}

Herein, we have highlighted the potential of LI-TOFMS for the comprehensive analysis of metalloporphyrins, where information about the component metallic/nonmetallic elements, characteristic fragments, and intact molecules are acquired simultaneously. Alternative molecular and elemental information can be obtained by varying the buffer gas pressure. To avoid spectral complication, this technique would be best used for pure organic compounds. LI-MS can accomplish the identification of analytes primarily in the solid phase using solventand matrix-free sample preparation, which expedites chemical characterization of metalloporphyrins.

\section{Acknowledgements}

We gratefully acknowledge financial support from the National Science Foundation of China (no. 21027011) and Program for Changjiang Scholars and Innovative Research Team in University (IRT13036). This work has also been supported by NFFTBS (no. J1310024).

\section{Notes and references}

1 C. Monnereau, P. H. Ramos, A. B. C. Deutman, J. A. A. W. Elemans, R. J. M. Nolte and A. E. Rowan, J. Am. Chem. Soc., 2010, 132, 1529-1531.

2 J. P. Collman, L. Zeng and R. A. Decreau, Chem. Commun., 2003, 2974-2975.

3 M. J. Griffith, K. Sunahara, P. Wagner, K. Wagner, G. G. Wallace, D. L. Officer, A. Furube, R. Katoh, S. Mori and A. J. Mozer, Chem. Commun., 2012, 48, 4145-4162.

4 C.-H. Wu, T.-Y. Pan, S.-H. Hong, C.-L. Wang, H.-H. Kuo, Y.-Y. Chu, E. W.-G. Diau and C.-Y. Lin, Chem. Commun., 2012, 48, 4329-4331.

5 G. J. Shaw, J. M. E. Quirke and G. Eglinton, J. Chem. Soc., Perkin Trans. 1, 1978, 1655-1659. 
6 B. D. Beato, R. A. Yost and J. M. E. Quirke, Org. Mass Spectrom., 1989, 24, 875-884.

7 N. Srinivasan, C. A. Haney, J. S. Lindsey, W. Zhang and B. T. Chait, J. Porphyrins Phthalocyanines, 1999, 3, 283291.

8 T. Gozet, L. Huynh and D. K. Bohme, Int. J. Mass Spectrom., 2009, 279, 113-118.

9 M. A. Kaczorowska and H. J. Cooper, Chem. Commun., 2011, 47, 418-420.

10 E. Mishra, J. Worlinsky, C. Brückner and V. Ryzhov, J. Am. Soc. Mass Spectrom., 2014, 25, 18-29.

11 M. S. Killian, J.-F. Gnichwitz, A. Hirsch, P. Schmuki and J. Kunze, Langmuir, 2009, 26, 3531-3538.

12 J. L. Sessler and D. Seidel, Angew. Chem., Int. Ed., 2003, 42, 5134-5175.

13 G. J. Van Berkel, S. A. McLuckey and G. L. Glish, Anal. Chem., 1992, 64, 1586-1593.

14 Q. Yu, L. Chen, R. Huang, W. Hang, B. Huang and J. He, TrAC, Trends Anal. Chem., 2009, 28, 1174-1185.

15 W. Hang, J. Anal. At. Spectrom., 2005, 20, 301-307.

16 L. Chen, L. Lin, Q. Yu, X. Yan, W. Hang, J. He and B. Huang, J. Am. Soc. Mass Spectrom., 2009, 20, 1355-1358.
17 Y. Gao, Y. Lin, B. Zhang, D. Zou, M. He, B. Dong, W. Hang and B. Huang, Anal. Chem., 2013, 85, 4268-4272.

18 Q. Yu, L. Li, W. Hang, J. He and B. Huang, Anal. Chem., 2011, 83, 2403-2407.

19 Y. Lin, R. Xu, L. Li, W. Hang, J. He and B. Huang, J. Anal. At. Spectrom., 2011, 26, 1183-1190.

20 Y. Lin, Q. Yu, R. Huang, W. Hang, J. He and B. Huang, Spectrochim. Acta, Part B, 2009, 64, 1204-1211.

21 M. Karas and F. Hillenkamp, Anal. Chem., 1988, 60, 22992301.

22 M. A. Kaczorowska and H. J. Cooper, J. Am. Soc. Mass Spectrom., 2010, 21, 1398-1403.

23 M. R. Domingues, M. G. S. Marques, C. A. Alonso, M. G. M. S. Neves, J. A. S. Cavaleiro, A. J. Ferrer-Correia, O. Nemirovskiy and M. Gross, J. Am. Soc. Mass Spectrom., 2002, 13, 1427-1431.

24 V. Margetic, A. Pakulev, A. Stockhaus, M. Bolshov, K. Niemax and R. Hergenröder, Spectrochim. Acta, Part B, 2000, 55, 1771-1785.

25 G. P. Pinho, H. Schittenhelm, W. W. Duley, S. A. Schlueter, H. R. Jahani and R. E. Mueller, Appl. Surf. Sci., 1998, 127129, 983-987. 